UCRL-ID-133245

\title{
Gun Requirements to Achieve High Field Spheromaks
}

T.K. Fowler

March 4, 1999

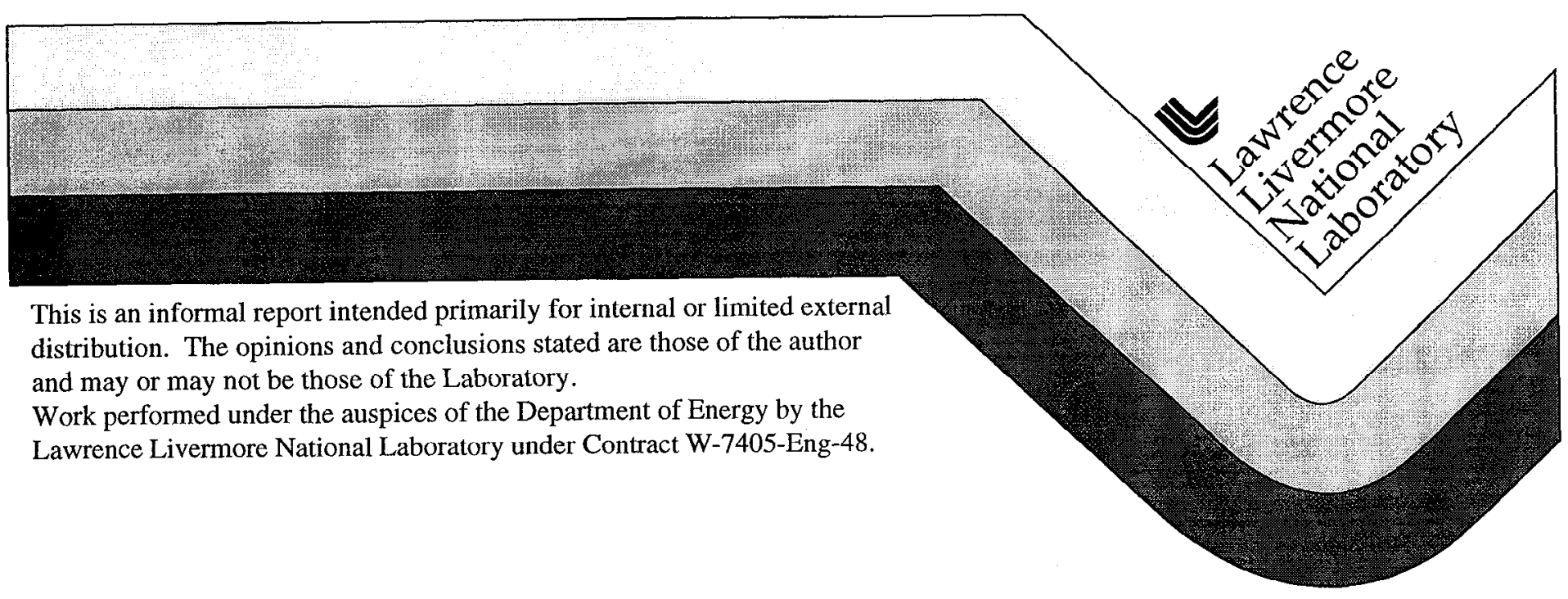




\section{DISCLAIMER}

This document was prepared as an account of work sponsored by an agency of the United States Government. Neither the United States Government nor the University of California nor any of their employees, makes any warranty, express or implied, or assumes any legal liability or responsibility for the accuracy, completeness, or usefulness of any information, apparatus, product, or process disclosed, or represents that its use would not infringe privately owned rights. Reference herein to any specific commercial product, process, or service by trade name, trademark, manufacturer, or otherwise, does not necessarily constitute or imply its endorsement, recommendation, or favoring by the United States Government or the University of California. The views and opinions of authors expressed herein do not necessarily state or reflect those of the United States Government or the University of California, and shall not be used for advertising or product endorsement purposes.

This report has been reproduced

directly from the best available copy.

Available to DOE and DOE contractors from the

Office of Scientific and Technical Information

P.O. Box 62, Oak Ridge, TN 37831

Prices available from (615) 576-8401, FTS 626-8401

Available to the public from the

National Technical Information Service

U.S. Department of Commerce

5285 Port Royal Rd.

Springfield, VA 22161 


\title{
GUN REQUIREMENTS TO ACHIEVE HIGH FIELD SPHEROMAKS
}

\author{
T. K. Fowler
}

February 1, 1999

\begin{abstract}
It is shown that a gun similar to that in the SSPX could demonstrate the high fields required for Pulsed Spheromak reactors merely by prolonging the pulse. Important considerations are choosing the voltage to exceed ohmic losses; designing the gun to avoid wasteful short-circuiting of current within the gun; and the injection efficiency factor, $f$, determined by the "sag" in the profile of $\lambda=\mu_{\mathrm{o}} \mathrm{j} / \mathrm{B}$. Typically $\mathrm{f}=0.75$ in experiments, giving an overall efficiency $>50 \%$ if short-circuiting is avoided. Theoretical transport models agree qualitatively with the need for a finite gradient in $\lambda$ to pump in helicity by current-driven tearing modes and suggest that pressure-driven resistive modes would not compete with current-driven modes during a buildup to ohmic ignition.
\end{abstract}

\section{Gun Operation}

Since small spheromak reactors require very high fields [1,2], demonstrating high fields using gun injection is the next important goal for the spheromak program. Slow injection of helicity is the key to creating high fields at low cost, which is the key to achieving ohmic ignition at low cost [1].

During slow gun injection a current I enters and exits the flux conserver following field lines generated externally by the gun solenoid with flux $\psi$, called the "flux core." The result is a screw pinch, initially stable. A spheromak is created when a screw pinch goes unstable inside a flux conserver, and the spheromak field continues to rise as long as power is fed into the screw pinch -- that is, into the "flux core." As current and helicity build up, Taylor relaxation both creates a closed spheromak confinement configuration and also guides the open-line flux core and gun current through the chamber [3]. When the current exceeds a threshold determined by $\psi$, instability and reconnection begin to form a closedline spheromak which gradually fills the volume while the flux core that feeds it shrinks to a skimny channel along the axis (see Figure 1, taken from work of Leaf Turner cited in Reference [3]).

The gun current is conveniently written in terms of the current density in the flux core of area $\psi / \mathrm{B}$, given by: 


$$
\mathrm{I}=\mathrm{j}(\psi / \mathrm{B})=\lambda(\mathrm{a}) \psi / \mu_{\mathrm{o}}=\mathrm{f} \lambda_{\mathrm{o}} \psi / \mu_{\mathrm{o}}
$$

where $f=\lambda_{o} \lambda(a)$ is the injection efficiency $[4]$ and $\lambda=\mu_{\mathrm{j}} \mathrm{j} / \mathrm{B}, \lambda(\mathrm{a})$ being the value in the flux core and $\lambda_{\mathrm{o}}$ being the lowest Taylor eigenvalue. As the spheromak begins to fill the volume, $\lambda$-- initially less than $\lambda_{\mathrm{o}}-$ saturates at $\lambda_{\mathrm{o}}$. After that, $\mathrm{j}$ and $\mathrm{B}$ continue to increase but $\mathrm{j} / \mathrm{B}=\lambda / \mu_{\mathrm{o}}$ is constant in the spheromak. Ideally unstable flow of magnetic energy out of the flux core causes $\lambda$ in the flux core to track that in the spheromak, giving $f=1$ at saturation (as was assumed in Figure 1). In fact $\lambda$ always sags significantly, inward during injection and outward during decay [4]. We shall characterize the sag in $\lambda$ by the parameter $\alpha$ used by Jarboe to characterize experimental results [4], given here by:

$$
-\alpha=\left(\lambda(a)-\lambda_{0}\right) / \lambda_{o}=f^{-1}-1 .
$$

Typical values inferred from magnetic probe measurements give $\alpha=-0.3$ during injection [4]. Even so the free energy -- the difference relative to the lowest Taylor state at a given helicity -- is of order $\alpha$, hence small enough to justify taking the Taylor state as representative of the spheromak; the actual free energy is $2 \%$ for $\alpha=-0.3$ [4]. From Eq. (3), $\alpha=-0.3$ corresponds to $f=0.75$, compared to Taylor's ideal value of unity, and the overall efficiency $f(1-\Delta V / V)>0.5$ for typical values, consistent with the assumption of 50\% efficiency in recent conceptual design studies for Pulsed Spheromak reactors [1]. We will return to the physics determining $\mathrm{f}$ in Section 3 .

\section{Gun Circuit -- Choosing the Voltage}

Since helicity is better conserved than energy, the fundamental "circuit" equation is that for helicity injection, given by $[3,4]$ : 


$$
\mathrm{dK} / \mathrm{dt}=2 \mathrm{~V} \psi-\mathrm{K} / \mathrm{t}_{\mathrm{a}}
$$

Initially, as the gun current I builds up from zero, $\mathrm{K}=2 \mathrm{LI} \psi$ (the product of the gun poloidal flux and toroidal flux due to I). Substituting this value of K into Eq. (3) and canceling $\psi$ gives the usual inductive circuit equation:

$$
\mathrm{Ld} / \mathrm{dt} \quad=\mathrm{V}-\mathrm{IR}_{\mathbf{Q}}
$$

with inductance $L=\mu R / 2$ and resistance $R=L / t_{\Omega}$ where $t_{\Omega}$ is the time constant for ohmic dissipation.

Instability sets in when the magnetic energy due to the current sufficiently exceeds the vacuum field energy due to $\psi$. By then most of the helicity is being deposited in the lowest Taylor state [4], the corresponding magnetic energy being $\mathrm{E}_{\mathrm{mag}}=\int \mathrm{B}^{2} / 2 \mu_{\mathrm{o}}$ $=\left(\lambda_{\mathrm{o}} / 2 \mu_{\mathrm{o}}\right) \mathrm{K}$. Multiplying Eq. (1) by $\left(\lambda_{\mathrm{o}} / 2 \mu_{\mathrm{o}}\right)$ gives the appropriate circuit equation valid during buildup and during steady state or field decay:

$$
\mathrm{dE}_{\mathrm{mag}} / \mathrm{dt}=\mathrm{fI}(\mathrm{V}-\Delta \mathrm{V})-\mathrm{P}_{\mathrm{Q}}-\mathrm{E}_{\mathrm{mag}} / \mathrm{t}_{\mathrm{fc}}
$$

Here we have separated the resistive loss into that due to the open-line flux core, included in $\Delta \mathrm{V}$; that due to the flux conserver with time constant $\mathrm{t}_{f c}$; and that due to the closed-line spheromak confinement volume. Note that the left side of Eq. (5) can, like the left side of Eq. (4), be interpreted as an "inductance" with $\mathrm{E}_{\mathrm{mag}}=\mathrm{LI}^{2} / 2$ if $\mathrm{L}$ is understood to be its geometric value multiplied by $\mathrm{K} / \psi^{2}$ [3]. We treat $\mathrm{V}$ as given (the generator $\mathrm{EMF}$ or capacitor bank Q/C, etc.) and assume that the power source is capable of delivering the current implied by our circuit equations.

First ignoring ohmic losses, we choose the power IV to build up the field in a reasonable time. Since I saturates, at constant $V$ the power is constant, the buildup time being: 


$$
t_{r I s c}=E_{m a g} / P_{n e t}=B^{2} R^{3} / P_{n c t}(M W)
$$

where $\mathrm{R}$ is the flux conserver radius, $\mathrm{P}_{\mathrm{net}}=\mathrm{fI}(\mathrm{V}-\Delta \mathrm{V})$ and approximately $\mathrm{E}_{\mathrm{mag}}=$ $B^{2} R^{3}$ in MJ [5]. For our reactor design, $P_{n \text { et }}=650 \mathrm{MW}$ giving a rise time of $250 \mathrm{~ms}$ to reach $B=27 \mathrm{~T}[1]$, to be compared with IV $=750 \mathrm{MW}$ in SSPX for a duration of milliseconds [6]. Next we ask whether these power levels can overcome ohmic losses. The spheromak ohmic loss is given by:

$P=\int_{\text {closed }} \eta j^{2}=E_{\text {mag }} / t_{\Omega}($ closed $)=\left(B^{2} R^{3} / 0.5 R^{2} T_{E}^{1 / 2}\right) M W$,

where on the right we evaluate $t_{\Omega}$ using the formula in Reference [5] for a parabolic temperature profile. Assuming electron heat conduction on open lines, we can evaluate the edge temperature $\mathrm{T}_{\mathrm{E}}$ (just that in the flux core) as:

$$
\mathrm{T}_{E}=0.04(\mathrm{~B} / \mathrm{f})^{2 / 5},
$$

where the $B$ scaling arises from $\chi n T_{E} / R^{2}=T_{E}{ }^{7 / 2} / R^{2}=\eta j^{2}$ with $j$ expressed in terms of $f, \lambda_{o}$ and $B$, with $\lambda_{0}=5 / R$. The core temperature $T$ is obtained by noting that ohmic heating gives roughly constant beta during buildup [5], giving:

$$
\mathrm{T}=\mathrm{T}_{\mathrm{E}}+12.5 \beta \mathrm{B}^{2} / \mathrm{n}_{20},
$$

where $\mathrm{n}_{20}$ is the density in units of $10^{20} \mathrm{~m}^{-3}$ and the second term represents temperature buildup on closed lines after reconnection. All temperatures are in $\mathrm{KeV}$ units. Combining Eqs. (7) - (9) gives, with typical $\beta=0.02$ during buildup:

$$
\mathrm{P}_{\Omega}=250 \mathrm{RB}^{7 / 5} \mathrm{f}^{3 / 5} /\left(1+6.25 \mathrm{~B}^{8 / 5} \mathrm{f}^{2 / 5} / \mathrm{n}_{20}\right) \quad \mathrm{MW}
$$

To buildup the field we require that the gun power IV $>P_{Q}$. Reference [2] shows that $\mathrm{P}_{\mathrm{Q}}$ is negligible if high field buildup is initiated at about the parameters expected to be 
achieved in SSPX ( $B=1 \mathrm{~T}$, flux core temperature $\left.T_{E}=0.1 \mathrm{KeV}\right)$. Estimates for earlier times (and hence SSPX performance) can be obtained from Eq. (10), given our assumption of a parabolic profile. Actual losses may be less if the profile is flatter and in any case IV $\gg P_{\Omega}$ is not difficult to satisfy by Eq. (10) if the density is not too largc. If desired the earliest reconnection stage can be accomplished quickly by providing, as in SSPX, an auxiliary fast bank to establish a closed line configuration [6].

In addition to the requirement on power, there is also a specific requirement on gun voltage in order that $\mathrm{V} \gg \Delta \mathrm{V}$, where $\Delta \mathrm{V}$ includes both the impedance in the power source and resistance in the flux core. Using Eq. (8), $\Delta \mathrm{V}$ due to the flux core resistance is given by:

$$
\Delta V=I(\eta \mathrm{L} / \mathrm{A})=0.16 \mathrm{~B} / \mathrm{fT}_{\mathrm{E}}{ }^{3 / 2}=20(\mathrm{~B} / \mathrm{f})^{2 / 5},
$$

where $I$ is taken from Eq. (1), $A=\psi / B, \eta=4 \times 10^{-8} / T_{E}{ }^{3 / 2}$ and we take length $L=R$ through the flux core.

From Eq. (11), we see that $\Delta \mathrm{V}$ never exceeds 100 volts even at reactor level fields $(B=27 \mathrm{~T}[2], f=0.75)$, to be compared with $V=5000$ volts in SSPX, which is therefore adequate for a reactor [6]. Hence the SSPX is a valuable step toward a high field experiment, the main difference being the pulse duration ( milliseconds in SSPX compared to $250 \mathrm{~ms}$ in our high field reactor design [1]). A high-field experiment could be carried out on SSPX itself up to limits on the strength of the flux conserver, if additional power could be provided to extend the pulse. Or a new flux conserver facility could be adapted to existing power supplies elsewhere.

\section{Gun Efficiency}

The physical origin of the sag in $\lambda$, reflected in a gun efficiency $f<1$, gets at the fundamental conundrum posed by Taylor's theory of relaxation, namely, the fact that $\lambda^{\prime}=0$ for the Taylor state, and the pressure is zero, also. By contrast, experiments do yield a finite pressure and $\lambda$ always sags inward or outward, implying a persistent, substantial free energy whereas the Taylor hypothesis assumes the free energy is dissipated to zero. 
As al ready noted, the observed sag in $\lambda$ corresponds typically to $2 \%$ of the magnetic energy during injection and perhaps $6 \%$ in the decay phase [4]. This is not at all consistent with observations based on heat confinement in CTX that time-averaged fluctuations involved in heat transport during field decay are many orders of magnitude weaker than this (a time-averaged "free energy" of order $\mathrm{S}^{-1}=10^{-1}$ in experiments) [7].

A possible explanation lies in the general theory of mode relaxation developed by Berk and Breizman to explain bursting and continuous turbulence in TAE modes [8]. Whether bursting or continuous, global transport occurs only if separated resonances overlap, which requires a finite free energy. By analogy, resistive MHD turbulence should inject helicity only if the available free energy is sufficient to maintain island overlap during transport, giving the condition:

$$
w^{2}\left[\left(\lambda^{\prime} / \lambda\right)^{2}+\beta\left(p^{\prime} / p\right)^{2}\right]>\delta B^{2} / B^{2}=\left[w^{4} k_{\theta}{ }^{2} / L_{s}{ }^{2} q^{2}\right],
$$

where the left side is the approximate free energy bound for both magnetic energy and pressure-driven modes, and on the right we have expressed the perturbation $\mathrm{SB}$ in terms of the island width $w$ by the usual formula [9], $\mathrm{L}_{\mathrm{S}}=\mathrm{q} / \mathrm{q}^{\prime}$ being the shear length and $\mathrm{k}_{\theta}$ the poloidal wavenumber. Global transport requires $w=w_{\max }$ (overlap) in Eq. (12), giving:

$$
\lambda^{\prime} / \lambda \quad\left(\text { or } \beta^{1 / 2} p^{\prime} / p\right)>w_{\max } k_{\theta} / L_{s} q
$$

In terms of $\mathrm{f}$ and Jarboe's parameter $\alpha, \lambda^{\prime} \mathrm{a} / \lambda=-2 \alpha=2\left(\mathrm{f}^{-1}-1\right)$.

We speculate that this condition for overlap, Eq. (13), rather than zero free energy, is the proper "angle of repose." While this equation does not easily give quantitative answers, it makes plausible the requirement for a finite sag in $\lambda$ in order to drive helicity during injection. Also it suggests that changes in $\mathrm{f}$ during injection would reflect changes in mode structure -- profiles -- rather than an explicit dependence on field or temperature.

Note that, since a finite $\lambda^{\prime}$ is implied, similarly a finite pressure gradient would be required to drive helicity by pressure-driven resistive modes, even though for that case the angle of repose for zero free energy is zero pressure. On this basis, because of the 
factor $\beta$, pressure-driven modes should be no more threat than magnetic modes during the buildup to ohmic ignition, during which typically $\beta<0.02-0.04$.

Finally, the relatively weak transport of helicity in general and the weak transport of heat in the decay phase of CTX can be reconciled with a large free energy through a duty cycle for overlap, denoted by $\mathrm{X}$, giving on time-average:

$$
<\delta \mathrm{B}^{2}>\quad=\delta \mathrm{B}^{2} \mathrm{X},
$$

where $\delta \mathrm{B}^{2}$ is the maximum allowed by the free energy (the left-hand side of Eq. (12)).

For resistive modes, Mattor [10] finds X to be bounded by:

$$
\mathrm{S}^{-1}<\mathrm{X}<\mathrm{S}^{-1 / 2}
$$

Here the upper bound represents continuous resistive turbulence while the lower bound is the "sawtooth" regime in which the transport rate is relatively independent of the free energy and depends instead on the ratio of the duration of overlap ( $t_{A}$, the Alfven time) and the growth time $t_{\Omega}$ (the resistive growth time) [10]. This ratio is just $S^{-1}$, which would account for the weak heat transport during the decay mode of CTX [7]. A larger value of X is needed to explain helicity transport during injection. Assuming $X=\mathrm{S}^{-1}$ gives a power flow $P<v_{A} B^{2} / S \mu_{o}=a B^{2} / t_{\Omega}$ even at $\delta B=B(100 \%$ free energy), which is inadequate to pump in power fast enough to outrun ohmic losses, as is required in injection. Most likely, given enough free energy for overlap, the system can always hover between sawtoothing and the continuum just so as to produce the time-averaged transport required under the circumstances. This is the assumption we have made in our transport model, calibrated to CTX [5] and applied to our reactor scenarios [1,2], in which we calculate the $<\delta \mathrm{B}^{2}>$ required to mainly roughly the Taylor state and then apply this $<\delta \mathrm{B}^{2}>$ to calculate Rechester-Rosenbluth heat transport.

Quantitative answers require detailed resistive MHD simulation. However, transport theory would be useful in interpreting simulation results as Berk and Breizman have already demonstrated for TAE modes [8]. In particular they have produced a modified quasilinear code that accurately reproduces non-linear simulations [11]. Weening and 
Boozer formulated a similar model for magnetic helicity transport, using hyper-resistive transport due to momentum diffusion [9].

\section{Short-Circuit in the Gun and Other Issues}

As described by Taylor [3], in the idealized "gun" in Figure 1 voltage is applied to an insulated polar cap on axis located at the pole to the left. The external bias flux in the gun enters through the polar cap on the left and exits at the other pole, guided into and out of the flux conserver by solenoids at each pole, the solenoid to the right serving as a divertor. The SSPX gun, sketched in Figure 2, differs mainly in the fact that the insulator is recessed to the rear of a coaxial gun structure based on the Marshall gun. The flux core is represented by field lines surrounding the spheromak, calculated by assuming a $\lambda$ profile with a sag similar to past experiments [6]. For this calculation the divertor coil is not energized so that vacuum field lines enter and exit inside the gun barrel, never entering the flux conserver. With current present, the flux core, which must follow the vacuum field at the entry and exit points, winds through the flux conserver to return along the wall to the gun where the vacuum field exits. Actually the current probably returns mainly through the copper flux conserver, due to field lines soaking into the copper.

Practical considerations giving rise to this design include a desire to avoid field lines exiting through the flux conserver wall -- a possible source of impurity in the confinement volume - and the need to reduce the heat load on the gun due to heat flow along open lines. Another important issue is short-circuiting in the gun.

Calculations for the SSPX design have shown that, in the configuration of Figure 2 in which the divertor coil and other auxiliary coils that could guide the bias flux are not activated, a sagging $\lambda$ gives efficient injection while complete relaxation to the Taylor state of constant $\lambda$ causes a large fraction of the gun current to short-circuit directly across the gun, never entering the flux conserver [12]. Activating the divertor coils helps considerably.

Given the uncertainty in how the $\lambda$ profile will evolve in new regimes beyond present experience, calculations such as those cited above give warning that care must be exercised to design guns to accommodate a range of $\lambda$ profiles without short-circuiting, while also addressing impurity and heat load issues. These issues, all the more important in 
designing a gun for slow buildup to high fields, can be addressed computationally as they have been for the SSPX design.

The heat load, mainly a concern during buildup when the flux core must be attached to the gun, is a fraction $F$ of $E_{m a g}$ (determined by $\Delta V$ and $P_{\Omega}$ ), while the area of deposition is determined by $\psi$ and the bias field $\mathrm{B}_{\mathrm{g} u \mathrm{a}}$. To avoid overheating we require that the temperature rise $\Delta \mathrm{T}$ (in degrees Kelvin) not be too large, where, uncooled:

$$
\Delta \mathrm{T}=\mathrm{FE} \mathrm{E}_{\mathrm{mag}} /\left(\mathrm{C}_{\mathrm{v}} \mathrm{d}(\psi / \mathrm{B})\right)
$$

where $C_{v}=3.3 \mathrm{MJ} / \mathrm{m}^{3} \mathrm{~K}$ is the volume heat capacity of copper of thickness $\mathrm{d}$.

\section{High Field Proof-of-Principle Experiment}

We have concluded that, given a sufficient gun voltage, slow gun injection can drive a flux conserver to any desired level of field $\mathrm{B}$, if the gun power is maintained for a sufficiently long time. The main design constraints relating the desired field $B$, rise time $t_{r I s c}$, and flux conserver radius $R$ to the gun current $I$, voltage $V$, and bias flux are:

$$
\begin{aligned}
& \mathrm{I}=\mathrm{f}\left(\lambda_{\mathrm{o}} \psi / \mu_{\mathrm{o}}\right)=\mathrm{f}(4 \psi / \mathrm{R}) \mathrm{MA} \\
& \mathrm{t}_{\mathrm{rIse}}=\mathrm{E}_{\mathrm{mag}} / \mathrm{IV}=\mathrm{B}^{2} \mathrm{R}^{3} / \mathrm{I}_{\mathrm{MA}} \mathrm{V} \\
& \mathrm{V}>\Delta \mathrm{V}=0.02(\mathrm{~B} / \mathrm{f})^{2 / 5} \mathrm{kV},
\end{aligned}
$$

taken from Eqs. (1), (6) and (11) respectively. The efficiency $f=0.75$ based on experimental results to date, if short-circuiting is avoided, as discussed in Section 4. Additional criteria are IV $>P_{\Omega}$ given by Eq. (11), which restricts the density during buildup, and Eq. (16), concerning the heat load.

Example parameters for a high field experiment, $\mathrm{R}=0.3 \mathrm{~m}$, are given in Table 1, together with reactor parameters. For both cases the required gun is similar to that in SSPX, operated for a much longer time ( $t_{\mathrm{r} I \mathrm{~s}}$ versus milliseconds in SSPX). 
A high field experiment would pave the way for an ignition experiment and eventually nuclear engineering tests with liquid walls.

Table 1. Example Parameters

$\begin{array}{llll} & \text { SSPX } & \text { POP } & \text { Reactor } \\ \text { B max (T) } & 1.0 & 27 & 27 \\ \mathrm{I}(\mathrm{MA}) & 0.15 & 0.15 / 0.8 & 0.2 / 1.0 \\ \mathrm{~V}(\mathrm{kV}) & 5 & 5 / 1 & 5 / 1 \\ \mathrm{t} \text { rise }(\mathrm{ms}) & <1 & 35 & 250 \\ \mathrm{R}(\mathrm{m}) & 0.5 & 0.3 & 0.6 \\ \psi(\text { weber }) & <0.03 & 0.01 / 0.05 & 0.02 / 0.1 \\ \Delta V \max (\mathrm{kV}) & 0.025 & 0.1 & 0.1\end{array}$

References

[1] T. K. Fowler and Per F. Peterson, "Conceptual Design of a Pulsed Spheromak Fusion Power Plant," UC-BFE-052, Nov. 5, 1998.

[2] T. K. Fowler, D. D. Hua, E. B. Hooper, R. W. Moir and L. D. Pearlstein, submitted for publication in Comments Plasma Phys. Contr. Fus. Oct.,1998.

[3] J. B. Taylor, Rev. Mod. Phys. 58, 741 (1986).

[4] T. R. Jarboe, Plasma Phys. Controlled Fusion 36, 945 (1994).

[5] T. K. Fowler and D. D. Hua, J. Fusion Energy 14, 181 (1995).

[6] E. B. Hooper et al, "Sustained Spheromak Physics Experiment," 17th IAEA Fusion Energy Conference, Yokohama, Japan, October 1998; to be published.

[7] E. B. Hooper, J.H. Hammer, C. W. Barnes and F. J. Wysocki, Fus. Tech. 29, 191 (1996).

[8] H. L. Berk and B. N. Breizman, "Overview of Nonlinear Theory of Kinetically Driven Instabilities," University of Texas Institute for Fusion Studies report, IFSR \# 841, September, 1998.

[9] R. H. Weening and A. H. Boozer, Phys. Fluids B 4, 159 (1992).

[10] N. Mattor, Phys. Plasmas 3, 1578 (1996).

[11] H. L. Berk, B. N. Breizman and J. Fitzpatrick, Nucl. Fusion 35, 1561 (1995).

[12] E. B. Hooper and L. D. Pearlstein, private communication. 

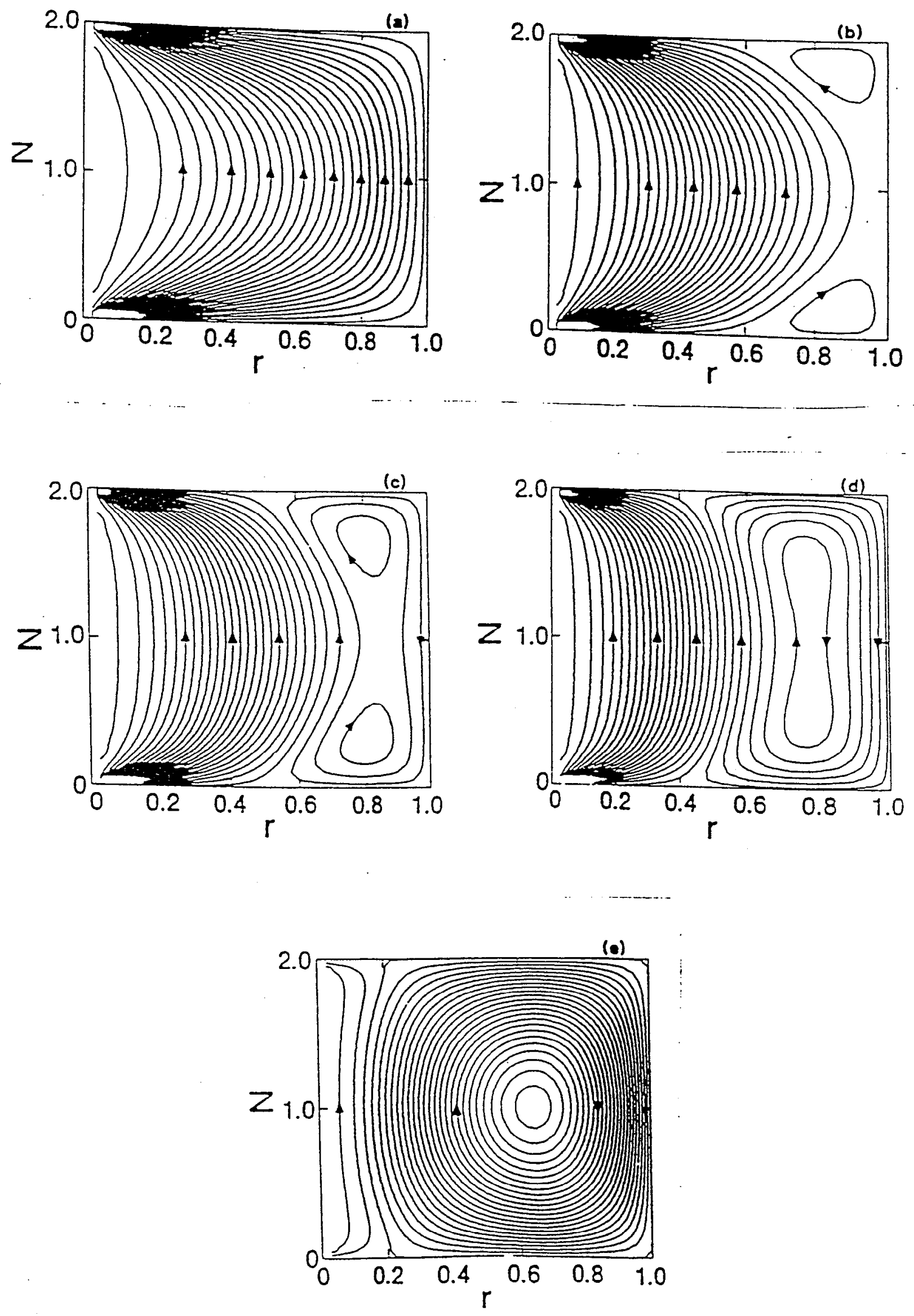

$$
\text { Figure } 1
$$




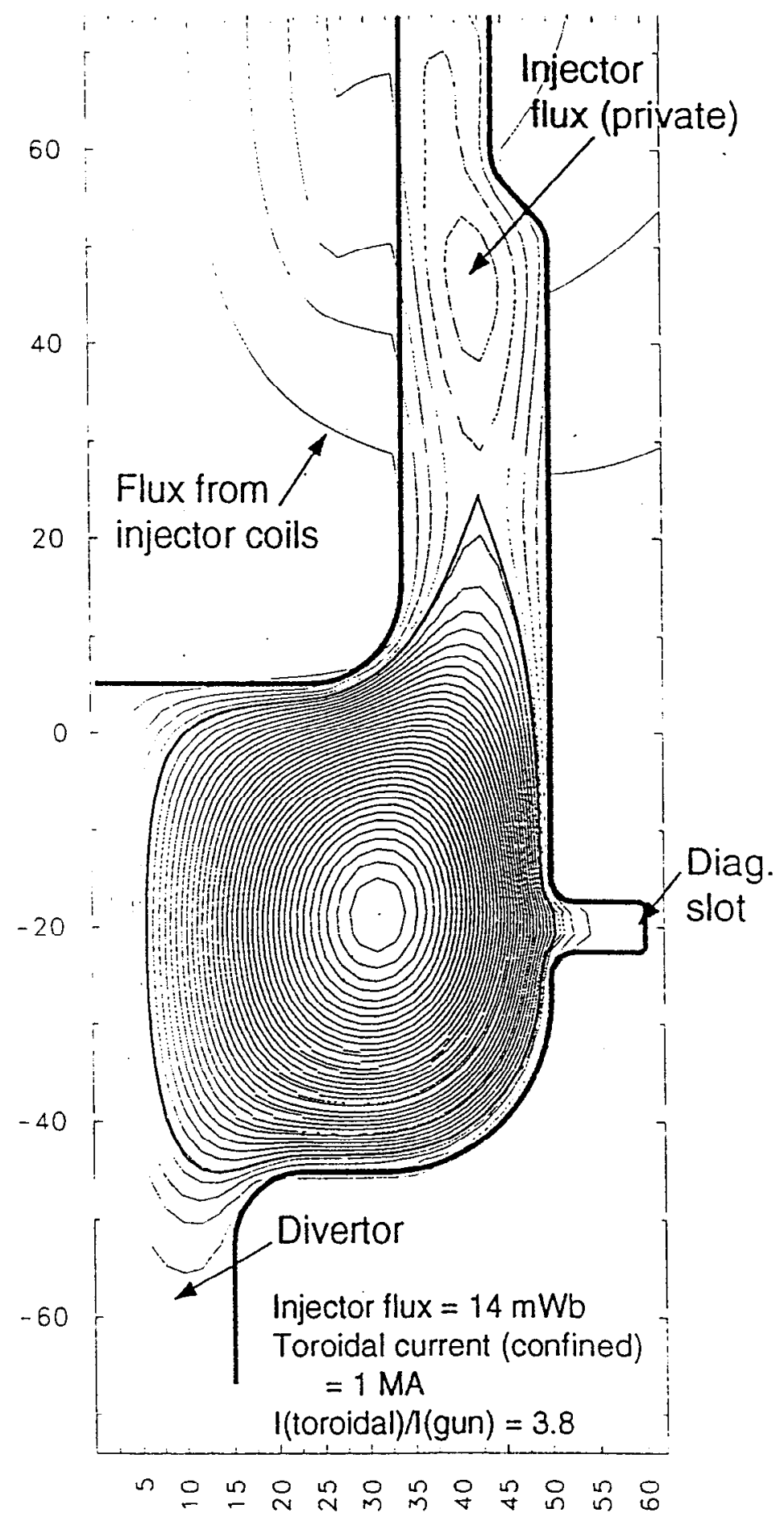

Figure 2 